\section{Estado de salud periodontal de pacientes trasplantados renales y calidad de vida asociada. Estudio exploratorio}

\author{
Periodontal health of renal patients and \\ quality of life associated. Exploratory study
}

\section{Resumen}

Objetivo. Determinar el estado de salud periodontal del paciente trasplantado renal, mediante la revisión de las historias clínicas diligenciadas por odontólogos generales o especialistas así como por la percepción de calidad de vida post-transplante, en relación con la salud bucal. Métodos. Estudio descriptivo transversal con 30 participantes de una unidad de transplante renal de la ciudad de Medellín, Colombia. Se exploraron antecedentes médico-odontológicos personales y familiares, examen bucal y periodontograma completo, por un solo examinador (Kappa $=0,79)$. Se aplicó la encuesta Oral Health Impact Profile (OHIP-14). Resultados. Edad promedio de 35 años (d.s $\pm 20,5), 50 \%$ hombres y $70 \%$ residentes en Medellín. El 46,7\% tuvo falla renal de carácter idiopático. Del $44,3 \%$ que presentaron problemas periodontales, el $73 \%$ tuvo periodontitis localizada, 31,8\% bolsas periodontales de $4 \mathrm{~mm}$. El cuestionario de calidad de vida aplicado mostró dos dimensiones afectadas: dolor físico y malestar psicológico (23,4\% y 20\% respectivamente). Conclusiones. Los pacientes trasplantados renales tuvieron compromiso periodontal. La severidad medida por la pérdida de inserción, mostró un resultado poco satisfactorio, considerando que los pacientes deben estar libres de factores irritativos e inflamatorios en la boca. El OHIP-14 es un instrumento amigable y sencillo para valorar la calidad de vida asociada a salud bucal.

Palabras clave: Enfermedades periodontales; Inmunosupresores; Calidad de vida; Trasplante de riñón; Enfermedad renal crónica (fuente: DeCS BIREME).

\begin{abstract}
Objective. Determine the periodontal health status of the renal transplantation patient, by reviewing the medical records filled out by general dentists or specialists as well as the perception of post-transplantation quality of life, in relation to oral health. Methods. Cross-sectional descriptive study with 30 participants from renal transplantation unit in the city of Medellín, Colombia. Personal and family medical-dental history, oral exam and complete periodontogram were explored by a single examiner (Kappa $=0.79)$. The Oral Health Impact Profile (OHIP-14) survey was applied. Results. Average age: 35
\end{abstract}

\section{Artículo Original}

\author{
Steven Betancur-Quintero 1,a, Sebastián Buitrago-Vásquez 1,a, \\ Jorge Armando Londoño-Grajales ${ }^{1, a}$, \\ William Londoño-Cuello 1,a, Cecilia María Martínez-Delgado 1,a, \\ Gustavo Adolfo Zuluaga-Valencia ${ }^{2, b}$ \\ ${ }^{1}$ Universidad de Antioquia, Facultad de Odontología, \\ Medellín, Colombia. \\ ${ }^{2}$ Hospital Pablo Tobón Uribe, Medellín, Colombia. \\ a Cirujano Dentista. \\ ${ }^{b}$ Médico Internista Nefrólogo.

\section{Correspondencia:} \\ Cecilia María Martínez Delgado: cmariamar@hotmail.com \\ Hospital Pablo Tobón Uribe. Unidad de Nefrología. Calle \\ 78 B NO. 69 - 240, Medellín, Colombia. \\ ORCID: 0000-0001-8409-7337
}

\section{Coautores:}

Steven Betancur Quintero

stevenbeta@hotmail.com

Sebastián Buitrago Vásquez sebasbuitra@gmail.com

Jorge Armando Londoño Grajales jorgelondono9016@hotmail.com

William Londoño Cuello

williamlon89@hotmail.com

Gustavo Adolfo Zuluaga Valencia

avoz01@yahoo.es

\section{Editor:}

Donald Ramos-Perfecto

Universidad Nacional Mayor de San Marcos, Perú.

Conflicto de intereses: los autores declaran no tener conflictos de interés.

Fuente de financiamiento: autofinanciado

Recibido: 06/10/19

Aceptado: $15 / 01 / 20$

Publicado: 24/02/20 
years (d.s \pm 20.5 ), 50\% men and 70\% residents in Medellín. 46.7\% had idiopathic renal failure. From the $44.3 \%$ who presented periodontal problems, $73 \%$ had localized periodontitis, $31.8 \%$ periodontal pockets of $4 \mathrm{~mm}$. The quality of life applied questionnaire showed two affected dimensions: physical pain and psychological distress $(23.4 \%$ and $20 \%$ respectively). Conclusions. The kidney transplantation patients had periodontal involvement. The severity measured by the loss of insertion, showed an unsatisfactory result, considering that patients should be free of irritating and inflammatory factors in the mouth. The OHIP-14 is a friendly and simple instrument to assess the quality of life associated with oral health.

Keywords: Periodontal diseases; Immunosuppressants; Quality of life; renal transplantation; Chronic kidney disease (source: MeSH NLM).

\section{Introducción}

La enfermedad renal crónica (ERC) es una enfermedad progresiva e inflamatoria que se caracteriza por la pérdida gradual y permanente de la función renal a largo plazo, como resultado de un espectro de enfermedades como diabetes, hipertensión, glomerulonefritis y trastornos autoinmunes ${ }^{1,2}$.

El trasplante renal "ofrece mejores resultados en términos de supervivencia, calidad de vida y menores costos" ${ }^{3}$. Esta condición de trasplantado hace más susceptible a los pacientes a determinadas infecciones; de esta manera, una salud bucal afectada, agrava los cuadros clínicos sistémicos, toda vez que ella (la salud bucal) está íntimamente ligada a la salud general

El tratamiento con inmunosupresores previene el rechazo agudo del órgano que se trasplanta ${ }^{4,5}$, pero puede incrementar la prevalencia y severidad de las enfermedades periodontales por el uso de dichos medicamentos, principalmente la ciclosporina ${ }^{6-14}$, factor condicionante para agrandamientos gingivales con la consecuente inflamación y retención bacteriana.

La prevalencia de enfermedad renal crónica (ERC) en el mundo, supera el $10 \%$, cifras semejantes al comportamiento de la diabetes ${ }^{15}$. Por otra parte, en Colombia un $31 \%$ de la población presenta bolsas periodontales compatibles con la presencia de periodontitis ${ }^{16}$, en pacientes sin compromiso renal, sin embargo no hay datos reportados en el país, sobre la prevalencia de periodontitis en pacientes con compromiso renal y/o con trasplante de riñón.

Actualmente se sugiere la existencia de una estrecha relación entre la enfermedad periodontal y el estado de salud general del paciente; lo que se consideraba un trastorno de tipo local, confinado a las encías, hoy se define como "inflamación sistémica con elevación de varios reactantes de fase aguda como la proteína $\mathrm{C}$ reactiva y el fibrinógeno" ${ }^{17}$.

Las enfermedades periodontales (EP) y la ERC presentan mecanismos patogénicos comunes, puesto que ambas afecciones se caracterizan por la presencia de un amplio espectro de citoquinas proinflamatorias ${ }^{17-24}$. El vínculo entre enfermedad periodontal y enfermedad renal crónica, puede deberse a la inflamación e infección concomitante que parecen ser causas importantes de morbilidad y mortalidad en los pacientes con ERC ${ }^{25-27}$.
Si hay enfermedades periodontales, hay mayor riesgo de desarrollar bacteriemia secundaria a diversos procedimientos dentales asociada con la gravedad de la inflamación periodontal ${ }^{28-30}$, generando complicaciones infecciosas sistémicas ${ }^{31-34}$ para estos pacientes, de tipo hiperrespuesta (que conduce a inflamación, daño tisular y reabsorción ósea) o hiporespuesta, lo que genera una progresión rápida de la periodontitis ${ }^{35-36}$.

Los pacientes con enfermedades crónicas, debilitantes e incapacitantes, pueden sentirse afectados en sus actividades cotidianas. Según la Organización Mundial de la Salud (OMS), la calidad de vida es "la percepción que un individuo tiene de su lugar en la existencia, en el contexto de la cultura y del sistema de valores en los que vive y en relación con sus objetivos, sus expectativas, sus normas, sus inquietudes. Se trata de un concepto que está influido por la salud física del sujeto, su estado psicológico, su nivel de independencia, sus relaciones sociales, así como su relación con su entorno" ${ }^{37}$.

Por las anteriores consideraciones, esta investigación se propuso determinar el estado de salud periodontal del paciente trasplantado renal, así como la percepción de calidad de vida postrasplante, en relación con la salud bucal.

\section{Métodos}

Estudio descriptivo transversal realizado en una unidad de trasplante renal de la ciudad de Medellín, aprobado por el Comité de Ética Institucional, según acta $\mathrm{N}^{\circ} 04$ de 2014. Se siguieron las recomendaciones de protección a la identidad de los encuestados, confidencialidad de los datos, normas de bioseguridad para los exámenes clínicos y manejo adecuado de desechos peligrosos. No se entrevistaron menores de edad.

Se revisaron historias clínicas que cumplieran con los siguientes criterios de inclusión: pacientes de ambos sexos, residentes en área urbana de Medellín y área metropolitana, edades entre 20 y 44 ańos, trasplantados renales exclusivamente con 3 años de evolución, sin otra patología asociada.

De un total de 72 participantes contactados telefónicamente entre octubre y diciembre de 2014, respondieron 30 pacientes al llamado, a quienes se les interrogó acerca de cuál antihipertensivo e inmunosupresor usaba cada paciente estudiado, por cuanto estos medicamentos están 
asociados a alteraciones periodontales; el examen bucal incluyó periodontograma completo utilizando la sonda OMS, posteriormente se aplicó la encuesta Perfil de Impacto de la Salud Oral (Health Impact Profile OHIP-14) validada en versión española.

Valoración de la salud bucal. Treinta pacientes fueron examinados clínicamente por un solo examinador estandarizado (Kappa= 0,79) en el índice de extensión y severidad ${ }^{15,37-39}$ clasificando la enfermedad periodontal de acuerdo a estándares establecidos por la Academia Americana de Periodontología (1999) ${ }^{38,40}$.

Valoración de la percepción de calidad de vida. Luego del examen periodontal se aplicó el cuestionario OHIP14 (validado por Montero-Martin et al., para la versión en español) ${ }^{41}$. Este cuestionario tiene 14 preguntas que valoran dimensiones funcionales y psicológicas de los problemas de salud bucal.

El cuestionario agrupa jerárquicamente dimensiones en el nivel biológico (deterioro), de comportamiento (limitación funcional, malestar y discapacidad) y, por último, social (discapacidad); por otra parte, es corto y fiable ${ }^{42}$.

Análisis estadístico. Para el análisis de los datos se utilizaron los programas Excel para Windows y SPSS 19.0 (Michigan Ill, Inc ${ }^{\circ}$ ). Se realizó primero un análisis descriptivo. Se exploró relación entre el índice de extensión y severidad con los resultados del índice de calidad de vida percibida, asumiendo que la variable condicionante fuese la calidad de vida percibida, mediante prueba de $\mathrm{Chi}^{2}$. Se trabajó con un nivel de significancia de 0,05.

\section{Resultados}

De una base de datos de 72 pacientes respondieron al llamado telefónico 30 pacientes, población relativamente joven (35 años $\pm 20,5)$. En cuanto al tiempo de ser receptores del trasplante, el $36,7 \%$ de los pacientes lo había recibido entre 1 y 2 años atrás. La falla renal más común fue de carácter idiopático (46,7\% de los casos); por causa definida, el reporte más frecuente fue el síndrome de Alport ( $20 \%$ de los casos), seguido de glomerulonefritis $(16,6 \%)$ y por accidente $(3,3 \%)$.

La medicación con inmunosupresores para lograr la estabilidad del órgano trasplantado y disminuir el riesgo de rechazo del mismo, se aplicó en el 100\% (30) de los pacientes entrevistados. En cada caso se pueden usar uno o varios inmunosupresores, para realizar una terapia exitosa (Tabla 1).

Para cada paciente se puede usar uno o varios antihipertensivos, cuya función es evitar la disfunción progresiva del órgano trasplantado y la mortalidad por enfermedad hipertensiva postrasplante (Tabla 2).

Estado de salud bucal. El 56,7\% de los pacientes estuvieron sanos para la condición periodontal; el 26,7\% presentó reacciones liquenoides, comprometiendo carrillos $(16,7 \%)$ y lengua $(13,3 \%)$. El estado periodontal indicó gingivitis generalizada (27\%) y periodontitis localizada (73\%); la severidad, valorada como profundidad al sondaje, arrojó que el $31,8 \%$ de los examinados tuvo bolsas periodontales de $4 \mathrm{~mm}$; no se encontraron bolsas de mayor profundidad. Ningún paciente sobrepasó el 30\% de extensión de la enfermedad, lo que indica enfermedad localizada.

Tabla 1. Características y frecuencia de uso de los inmunosupresores en pacientes trasplantados renales

\begin{tabular}{llcc}
\hline Inmunosupresor & Familia & Frecuencia absoluta (n) & Frecuencia relativa (\%) \\
\hline Prednisolona & Corticoides & 26 & 86,7 \\
Ciclosporina & Inmunosupresor undecapéptido & 10 & 33,3 \\
Tacrolimus & Macrólido & 20 & 66,6 \\
Sirolimus & Macrólido & 2 & 6,6 \\
Micofenolato & Inmunosupresor de última generación & 20 & 66,6 \\
Azatioprina & Derivado imidazólico & 4 & 13,3 \\
Betalacept & Proteína de fusión & 1 & 3,3 \\
Everolimus & Agentes Inmunosupresores ATC L04 & 2 & 6,6 \\
\hline
\end{tabular}

Tabla 2. Características y frecuencia de uso de antihipertensivos en pacientes trasplantados renales

\begin{tabular}{llcc}
\hline Antihipertensor & Familia & Frecuencia absoluta (n) & Frecuencia relativa (\%) \\
\hline Metoprolol & Beta-bloqueadores & 15 & 50 \\
Enalapril & Inhibidores ECA & 9 & 30 \\
Hidroclorotiazida & Diuréticos tiazídicos & 0 & 0 \\
Losartan & Antagonistas angiotensinas II & 4 & 13,3 \\
Clonidina & Antagonistas centrales & 2 & 6,6 \\
Amlodipina & Bloqueadores de los canales de calcio & 4 & 13,3 \\
Prazosine & Alfa-bloqueadores & 7 & 23,3 \\
\hline
\end{tabular}


La presencia de cálculos como factor irritativo local que puede agravar el cuadro clínico del paciente, se encontró en el $63,3 \%$ de los pacientes examinados.

Calidad de vida relacionada con salud bucal. En la Tabla 3, se muestran las distribuciones porcentuales de las diferentes preguntas que componen el instrumento OHIP-14 de los pacientes trasplantados renales. Para el caso de las dimensiones limitación funcional, incapacidad física, incapacidad psicológica, incapacidad social y minusvalía, los porcentajes se concentran mayoritariamente en las opciones nunca y casi nunca.

En la dimensión limitación funcional, el 96,7\% de los pacientes reportó que nunca o casi nunca presentaron compromiso de este componente. En la dimensión de dolor físico, el $26,7 \%$ de estos pacientes respondieron afirmativamente. En la dimensión malestar psicológico, el 6,7\% respondió que frecuentemente se ha visto comprometida. Los pacientes trasplantados nunca o casi nunca han tenido incapacidad social ni consideraron tener algún grado de minusvalía en relación con la salud bucal.
El instrumento aplicado (OHIP-14) entregó resultados satisfactorios que pueden traducirse en una calidad de vida percibida como positiva; las dimensiones con algún grado de compromiso fueron dolor físico y malestar psicológico.

Cuando se relacionó el índice de calidad de vida percibida con índice de extensión y severidad, no se halló significancia estadística $(\mathrm{p}>0,05)$.

\section{Discusión}

El estado de salud periodontal cobra mayor importancia al momento de tratar a los pacientes con afecciones sistémicas, porque se sugiere que la relación enfermedad renal crónica y enfermedades periodontales es bidireccional y que al controlar dichos factores inflamatorios, la salud de los pacientes mejora ${ }^{42,43}$.

Varios componentes pueden aumentar la susceptibilidad del paciente a sufrir ERC; el sexo, la edad, la presencia de enfermedades como diabetes mellitus e hipertensión,

Tabla 3. Distribución porcentual de las respuestas del OHIP-14 de los paciente trasplantados renales. Medellín, 2014

\begin{tabular}{|c|c|c|c|c|c|c|c|c|c|c|}
\hline \multirow{2}{*}{ Pregunta/dimensión $(n=30)$} & \multicolumn{2}{|c|}{ Nunca } & \multicolumn{2}{|c|}{ Casi nunca } & \multicolumn{2}{|c|}{ Ocasionalmente } & \multicolumn{2}{|c|}{ Frecuente } & \multicolumn{2}{|c|}{ Muy frecuente } \\
\hline & $\mathrm{n}$ & $\%$ & $\mathrm{n}$ & $\%$ & $\mathrm{n}$ & $\%$ & $\mathrm{n}$ & $\%$ & $\mathrm{n}$ & $\%$ \\
\hline \multicolumn{11}{|l|}{ Limitación funcional } \\
\hline $\begin{array}{l}\text { ¿Tiene usted incomodidad para pronunciar algunas palabras } \\
\text { debido a problemas con sus dientes, boca o prótesis dentales? }\end{array}$ & 29 & 96,6 & 1 & 3,4 & 0 & 0 & 0 & 0 & 0 & 0 \\
\hline $\begin{array}{l}\text { ¿Ha sentido usted que su sensación del gusto ha empeorado } \\
\text { debido a problemas con sus dientes, boca o prótesis dentales? }\end{array}$ & 29 & 96,6 & 0 & 0 & 0 & 0 & 1 & 3,4 & 0 & 0 \\
\hline \multicolumn{11}{|l|}{ Dolor físico } \\
\hline ¿Tiene usted alguna incomodidad dolorosa en su boca? & 15 & 50 & 8 & 26,7 & 2 & 6,7 & 4 & 13,3 & 1 & 3,4 \\
\hline $\begin{array}{l}\text { ¿Ha sentido usted incomodidad al comer algunos alimentos debi- } \\
\text { do a problemas con sus dientes, boca o prótesis dentales? }\end{array}$ & 16 & 53,3 & 8 & 26,6 & 3 & 10 & 3 & 10 & 0 & 0 \\
\hline \multicolumn{11}{|l|}{ Malestar psicológico } \\
\hline $\begin{array}{l}\text { ¿Ha estado usted preocupado debido a problemas con sus dien- } \\
\text { tes, boca o prótesis dentales? }\end{array}$ & 13 & 43,3 & 11 & 36,7 & 3 & 10 & 3 & 10 & 0 & 0 \\
\hline $\begin{array}{l}\text { ¿Se ha sentido nervioso debido a problemas con sus dientes, boca } \\
\text { o prótesis dentales? }\end{array}$ & 22 & 73,3 & 5 & 16,7 & 1 & 3,4 & 2 & 6,7 & 0 & 0 \\
\hline \multicolumn{11}{|l|}{ Incapacidad física } \\
\hline $\begin{array}{l}\text { ¿Ha sido su dieta poco satisfactoria debido a problemas con sus } \\
\text { dientes, boca o prótesis dentales? }\end{array}$ & 29 & 96,6 & 0 & 0 & 1 & 3,4 & 0 & 0 & 0 & 0 \\
\hline $\begin{array}{l}\text { ¿Ha tenido usted que interrumpir sus comidas debido a proble- } \\
\text { mas con sus dientes, boca o prótesis dentales? }\end{array}$ & 25 & 83,3 & 4 & 13,3 & 1 & 3,4 & 0 & 0 & 0 & 0 \\
\hline \multicolumn{11}{|l|}{ Incapacidad psicológica } \\
\hline $\begin{array}{l}\text { ¿Encuentra usted dificultad para relajarse debido a problemas } \\
\text { con sus dientes, boca o prótesis dentales? }\end{array}$ & 26 & 86,6 & 4 & 13,3 & 0 & 0 & 0 & 0 & 0 & 0 \\
\hline $\begin{array}{l}\text { ¿Se ha sentido usted perturbado frente a otras personas debido a } \\
\text { problemas con sus dientes, boca o prótesis dentales? }\end{array}$ & 24 & 80 & 6 & 20 & 0 & 0 & 0 & 0 & 0 & 0 \\
\hline \multicolumn{11}{|l|}{ Incapacidad social } \\
\hline $\begin{array}{l}\text { ¿Se ha sentido usted irritable con otras personas debido a proble- } \\
\text { mas con sus dientes, boca o prótesis dentales? }\end{array}$ & 27 & 90 & 3 & 10 & 0 & 0 & 0 & 0 & 0 & 0 \\
\hline $\begin{array}{l}\text { ¿Tiene usted dificultad de hacer sus trabajos habituales debido a } \\
\text { problemas con sus dientes, boca o prótesis dentales? }\end{array}$ & 30 & 100 & 0 & 0 & 0 & 0 & 0 & 0 & 0 & 0 \\
\hline \multicolumn{11}{|l|}{ Minusvalía } \\
\hline $\begin{array}{l}\text { ¿Ha sentido usted que su vida es, en general, menos satisfactoria, } \\
\text { debido a problemas con sus dientes, boca o prótesis dentales? }\end{array}$ & 28 & 93,3 & 2 & 6,7 & 0 & 0 & 0 & 0 & 0 & 0 \\
\hline $\begin{array}{l}\text { ¿Se ha sentido usted totalmente incapaz de funcionar debido a } \\
\text { problemas con sus dientes, boca o prótesis dentales? }\end{array}$ & 30 & 100 & 0 & 0 & 0 & 0 & 0 & 0 & 0 & 0 \\
\hline
\end{tabular}


pueden desencadenarla; así mismo, la edad aumenta la susceptibilidad a la ERC ${ }^{44}$.

Los resultados indicaron que la causa de falla renal más común fue la idiopática; un estudio realizado por Saldívar et al. ${ }^{45}$ reportó que las causas más comunes de ERC en pacientes menores de 30 años fueron glomerulonefritis, hipoplasia renal y preeclampsia y en los mayores de 40 años de edad, padecimientos crónico-degenerativos.

Los inmunosupresores y antihipertensivos empleados en este tipo de pacientes tienen el objetivo de prevenir y tratar el rechazo agudo y evitar la lesión crónica del injerto, minimizando los efectos adversos ${ }^{46,47}$. Un estudio realizado en México encontró que los inhibidores de calcineurina tienen una relación con la inflamación gingival o con el daño periodontal, mayormente la $\mathrm{Ci}$ closporina $\mathrm{A}^{48,49}$; este hallazgo es semejante al del presente estudio.

La periodontitis asociada a los malos hábitos de higiene bucal representa el mayor riesgo para personas sin compromiso sistémico, con mayor razón en pacientes inmunocomprometidos. Un estudio realizado en Croacia (2015) encontró que la hiperplasia gingival en encía marginal en los dientes anteriores superiores e inferiores fue un hallazgo frecuente ${ }^{50}$; otro estudio realizado en Serbia (2013) reportó que los pacientes trasplantados renales presentaron una reducción en los parámetros clínicos periodontales en relación con la duración de la terapia inmunosupresora ${ }^{51}$.

En el estudio realizado, la calidad de vida relacionada con la salud bucal no se percibió muy comprometida; la dimensión de dolor fue la que obtuvo mayor puntuación. Hay estudios que refieren que después del trasplante renal, por efecto de los fármacos se puede presentar disgeusia, xerostomía, petequias y alteraciones articulares ${ }^{6,52-54}$.

En un estudio realizado en Medellín (Colombia) por Posada et al., en una población sin nefropatías, los encuestados manifestaron un mayor compromiso de su calidad de vida, comprometiendo mucho más las dimensiones relacionadas con malestar psicológico y con dolor físico ${ }^{55}$.

Por tanto, los pacientes trasplantados renales del presente estudio tuvieron compromiso periodontal en su forma localizada, lo que pudiera agravar su condición sistémica. Y por otra parte, la calidad de vida medida a través del instrumento OHIP-14 mostró que es amigable y sencillo, porque permite a los pacientes entender de una mejor manera, lo que significa vivir con una enfermedad crónica.

\section{Agradecimientos}

A los pacientes de la Unidad de Trasplante Renal del Hospital Pablo Tobón Uribe y a la Facultad de Odontología de la Universidad de Antioquia.

\section{Referencias bibliográficas}

1. Rojas M P, Meza J, Ríos K. Manifestaciones bucales en pacientes con insuficiencia renal crónica en hemodiálisis. Rev Estomatol Herediana. 2014;24(3):147-154.

2. Navia M F, Muñoz E E, López O P. Relación del estado de salud bucal y condiciones en el paciente con enfermedad renal crónica en tratamiento. Rev Salud Pública. 2013;15(6):857-869.

3. Douthat W G, Fernández P, Rechene J, Chiurchiu C R, De Arteaga J, Massari P U. Trasplante renal y disminución de la mortalidad en los programas de diálisis crónica. Medicina. (B. Aires) 2014;74(1):1-8.

4. Bahamondes C, Godoy J. Cyclosporine-induced gingival hyperplasia: Report of one case. Rev Med Chile. 2007;135(3):370-374.

5. Pejcic A, Djordjevic V, Kojovic D, Zivkovic V. Effect of periodontal treatment in renal transplant recipients. Med Princ Pract. 2014;23:149-153.

6. Ismail G, Dumitriu HT, Dumitriu AS, Ismail FB. Periodontal disease: a covert source of inflammation in chronic kidney disease patients. Int J Nephrol. 2013, 2013:515796. DOI: 10.1155/2013/515796.

7. Castronovo G, Liani G, Fedon A, De Iudicibus S, Decorti $\mathrm{G}$, Costantinides $\mathrm{F}$ et al. The effect of nonsurgical periodontal treatment on the severity of drug-induced gingival overgrowth in transplant patients. Quintessence Int. 2014;45(2):115-124.

8. Zalba J I, Flichy A J. Empleo de probióticos en odontología. Nutr Hosp. 2013;28(Suppl 1):49-50.

9. Lindhe J, Niklaus P L, Thorkild K. Clinical Periodontology and Implant Dentistry. 5a ed. Bogotá. Médica Panamericana Editores, 2008.

10. Suzuki N, Yoneda M, Hirofuji T. Mixed Red-Complex Bacterial Infection in Periodontitis. Int J Dent [Internet]. 2013:587279 Consultado 8 mayo 2016] Disponible en: https://www.ncbi.nlm.nih.gov/pmc/articles/ PMC3606728/

11. Newman M G, Takey H H, Perry M S, Klokkevold M S, Carranza F A. Periodontología clínica. 10a ed. México. Mcgraw-Hill Editores, 2007.

12. Botero J E, Bedoya E. Determinants of Periodontal Diagnosis. Rev Clin Periodoncia Implantol Rehabil Oral . 2010;3(2):94-99.

13. Linden G J, Herzberg M C, Periodontitis and systemic diseases: a record of discussions of working group 4 of the Joint EFP/AAP Workshop on Periodontitis and Systemic Diseases. J Clin Periodontol. 2013;40(Suppl.14):S20-S23.

14. Birkan A, Kuru B E. Periodontal treatment in a generalized severe chronic periodontitis patient: A case report with 7-year follow-up. Eur J Dent. 2015;9(2):288-292.

15. James, MT, Hemmelgarn, BR, Tonelli M. Early recognition and prevention of chronic kidney disease. Lancet. 2010;375:1296-1309.

16. Ministerio de Salud de Colombia. IV Estudio Nacional de Salud Bucal (ENSAB-IV). Bogotá. República de Colombia, Ministerio de Salud. 2014. [Consultado el 23 
de setiembre 2018]. Accesible en: https://www.minsalud. gov.co/sites/rid/Lists/BibliotecaDigital/RIDE/VS/PP/ ENSAB-IV-Situacion-Bucal-Actual.pdf

17. González B, Pintó X, Salas E. Relationship between cardiovascular disease and dental pathology. Systematic review. Med Clín (Barc). 2017;149(5):211-216.

18. Gupta Sh, Gupta I, Gupta R, Gupta P. Role of C-Reactive protein in periodontal disease- a review. IJCMR 2017;4(5):980- 985.

19. Gupta J, Mitra N, Kanetsky P A, Devaney J, Wing M R, Reilly $\mathrm{M}$ et al. Association between albuminuria, kidney function, and inflammatory biomarker profile in CKD in CRIC. Clin J Am Soc Nephrol. 2012;7(12):19381946.

20. Carillo H P, Oliveira C, Medeiros M C, Vieira A P. Effect of non-surgical periodontal treatment on the subgingival microbiota of patients with chronic kidney disease. Braz Oral Res. 2012;26(4):366-72.

21. Bolla V, Kumari P S, Munnangi S R, Kumar D S, Durgabai Y. Evaluation of Serum C-reactive Protein Levels in Subjects with Aggressive and Chronic Periodontitis in Comparison with Healthy Controls: A Clinico-biochemical Study. Int J Appl Med Res. 2017;7(2):121-124.

22. Boutros E, Al Nashar A, Al Haji S, Ganem R. The relationship between Periodontal Disease Severity and Systemic Diseases: Retrospective study. IDJSR 2017;4(3):141143.

23. Linden G J, Lyons A, Scannapieco F A. Periodontal systemic associations: review of the evidence. J Periodontol. 2013;84(4 Suppl.):8-19.

24. Da Silva MK, de Carvalho ACG, Alves EHP, da Silva FRP, Pessoa LDS, Vasconcelos DFP. Genetic Factors and the Risk of Periodontitis Development: Findings from a Systematic Review Composed of 13 Studies of Meta-Analysis with 71,531 Participants. Int J Dent. 2017;2017:1914073. DOI: 10.1155/2017/1914073.

25. Sei Y, Mizuno M, Suzuki Y, Imai M, Higashide K, Harris CL, Sakata F et.al. Expression of membrane complement regulators, CD 46, CD55 and CD59, in mesothelial cells of patients on peritoneal dialysis therapy. Mol Immunol. 2015;65(2):302-309.

26. Belibasakis G N, Mylonakis E. Oral infections: clinical and biological perspectives. Virulence. 2015;6(3):173 176.

27. Scannapieco F A,Cantos A. Oral inflammation and infection, and chronic medical diseases: implications for the elderly. Periodontol 2000. 2016;72(1):153-75.

28. Tawfig A, Jamal B, Eskandrani R, AlDayel L, Sara AlTwaijry, AlGhamdi E, Ahmed B. Assessment of Periodontal Disease Severity among Patients at Different Stages of Chronic Kidney Disease. J Int Oral Health. 2016;8(3):307-312.

29. Chizyński A, Pawlicka H, Krzysztof C. Infective endocarditis prevention prior to dental procedures. J Stoma. 2012;65(5):729-738.

30. Horliana ACRT, Chambrone L, Foz AM, Artese HPC, Rabelo MDS, et al. Dissemination of Periodontal Pathogens in the Bloodstream after Periodontal Procedures: A Systematic Review. PLoS ONE [Internet]. 2014 [Citado el 18 febrero 2016]; 9(5):98271. Disponible en: http:// journals.plos.org/plosone/article?id=10.1371/journal. pone.0098271

31. Nguyen CM, Kim JWM, Quan VH, Nguyen BH, Tran SD. Periodontal associations in cardiovascular diseases: The latest evidence and understanding. J Oral Biol Craniofac Res. 2015;5(3):203-206.

32. Borgnakke W S. Does Treatment of Periodontal Disease Influence Systemic Disease? Dent Clin North Am. 2015;59(4):885-917.

33. Suliman N M, Johannessen A C, Ali R W, Salman H, Åstrøm A N. Influence of oral mucosal lesions and oral symptoms on oral health related quality of life in dermatological patients: a cross sectional study in Sudan. BMC Oral Health. 2012;12:12:19.

34. Carvalho A, ArmaniII F, ArapIII A, NejmII T, AndradeII J, BufarahII H, Syllos D. Relationship between periodontal disease and cardiovascular risk factors among young and middle-aged Brazilians. Cross-sectional study. Sao Paulo Med J. 2017;135(3):226-233.

35. James MT, Hemmelgarn BR, Tonelli M. Early recognition and prevention of chronic kidney disease. Lancet. 2010;375:1296-1309.

36. Grupo de la OMS sobre la calidad de vida. La gente y la salud. ¿Qué calidad de vida? Foro Mundial de Ia Salud. [Consultado el 12 noviembre 2017]; Accesible en: http://apps.who.int/iris/bitstream/10665/55264/1/ WHF_1996_17_n4_p385-387_spa.pdf

37. Ismail G, Traian H, Silvia A, Bahtiar F. Periodontal Disease: A Covert Source of Inflammation in Chronic Kidney Disease Patients. Int J Nephrol. 2013;2013:515796. DOI: $10.1155 / 2013 / 515796$.

38. Kim YJ, Moura LM, Caldas CP, Perozini C, Ruivo GF, Pallos D. Evaluation of periodontal condition and risk in patients with chronic kidney disease on hemodialysis. Einstein (Sao Paulo). 2017;15(2):173-177

39. Maya JC, Sánchez N, Posada A, Agudelo AA, Botero JE. Periodontal disease and its relationship with clinical and sociodemographic variables in adult patients treated in a service/teaching institution. Rev Odont Mex. 2017;21(3):160-167.

40. Montero J, Bravo M, Albaladejo A, Hernández LA, Rosel EM. Validation the Oral Health Impact Profile (OHIP-14 sp) for adults in Spain. Med Oral Patol Oral Cir Bucal. 2009;14(1):44-50.

41. Mello dos Santos C, de Oliveira BH, Nadanovsky P, Balbinot J, Keller R, Neves F. The Oral Health Impact Profile-14: a unidimensional scale? Cad. Saúde Pública. 2013;29(4):749-757.

42. AlJehani YA. Risk Factors of Periodontal Disease: Review of the Literature. Int J Dent. 2014;2014:182513. DOI: $10.1155 / 2014 / 182513$

43. Fernandez J, Barrionuevo P, Mastronardi CA. Periodontal Disease and Its Systemic Associated Diseases. Mediators Inflamm. 2015;2015:153074. DOI: $10.1155 / 2015 / 153074$.

44. Stevens PE, Levin A. For the Kidney Disease: Improving Global Outcomes Chronic Kidney Disease Guideline Development Work Group Members. Evaluation and mana- 
gement of chronic kidney disease: synopsis of the kidney disease: improving global outcomes 2012 clinical practice guideline. Ann Inter Med. 2013];158(11):825-830.

45. Ávila MN, Conchillos G, Rojas IC, rdoñez AE, Ramírez HJ. Enfermedad renal crónica: causa y prevalencia en la población del Hospital General La Perla. Med Int Mex. 2013;29(5):473-478.

46. Bestard O, Campistol JM, Morales JM, Sánchez A, Cabello M, Cabello V, et al. Avances en la inmunosupresión para el trasplante renal: Nuevas estrategias para preservar la función renal y reducir el riesgo cardiovascular. Nefrología 2012;32(3):374-384.

47. Ji-Yeun C, Jihyun Y, Ha ChB, Jaeseok Y, Sung K, , Chan $\mathrm{K}$, et al. Immunosuppressant prescription pattern and trend in kidney transplantation: A multicenter study in Korea. PLoS One [Internet]. 2017;12(8):0183826. [Citado el: 12 noviembre 2017]. Disponible en: https://doi. org/10.1371/journal.pone.0183826.

48. Gong Y, Bi W, Cao L, Yang Y, Chen J, Yu Y. Association of CD14-260 polymorphisms, red-complex periodontopathogens and gingival crevicular fluid cytokine levels with cyclosporine A-induced gingival overgrowth in renal transplant patients. J Periodontal Res. 2013;48(2):203-212.

49. Fu MM, Chin YT, Fu E, Chiu HC, Wang LY, Chiang CY, Tu HP. Role of Transforming Growth Factor-beta 1 in Cyclosporine-Induced Epithelial-to-Mesenchymal Transition in Gingival Epithelium. J Periodontol. 2015;86:120-128.
50. Gürkan A, Becerik S, Öztürk VÖ, Atmaca H, Atilla G, Emingil G. Interleukin-6 Family of Cytokines in Crevicular Fluid of Renal Transplant Recipients with and Without Cyclosporine A-Induced Gingival Overgrowth. J Periodontol. 2015; 86(9):1069-1077.

51. Gaspar M, Glavina A, Grubisic K, Sabol I, Busic M, Mravak-Stipetić M. The Oral Cavity State in Renal Transplant Recipients. Acta Stomatol Croat. 2015;49(3):204-213.

52. Pejcic A, Djordjevic V, Kojovic D, Zivkovic V, Minic I, Mirkovic D, et al. Effect of periodontal treatment in renal transplant recipients. Med Princ Pract. 2014;23(2):149-153.

53. de Souza CM, Braosi AP, Luczyszyn SM, Olandoski M, Kotanko P, Craig RG, et al. Association Between Oral Health Parameters, Periodontitis and its Treatment and Mortality in Hemodialysis Patients. J Periodontol. 2014;85(6):169-178.

54. Hernández C. Oral disorders in patients with chronic renal failure. Narrative review. J Oral Res. 2016;5(1):27-34.

55. Posada-Lopez A, Agudelo-Suarez A, Murillo-Pedrozo AM, Ramirez-Sepulveda KA et al. Oral health impact in quality of live in adults treated at the Faculty of Dentristy, University of Antioquia. Rev Fac Odontol Univ Antioq. 2014;25(suppl):96-108. 
